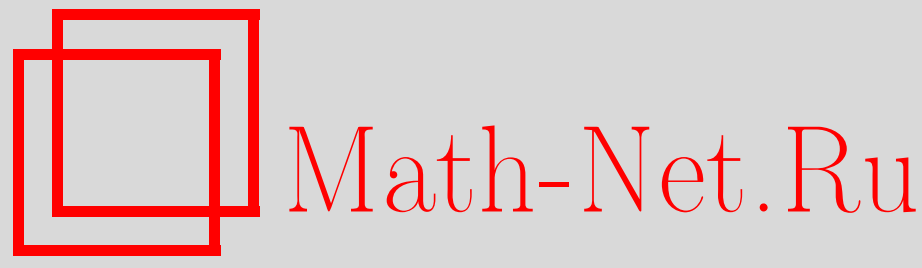

Обцероссийский математический портал

С. А. Шкарин, О спектрах линейных непрерывных операторов на сепарабельных пространствах Фреше, Матем. заметки, 2001, том 69, выпуск 4, 637-640

DOI: https://doi.org/10.4213/mzm693

Использование Общероссийского математического портала Math-Net.Ru подразумевает, что вы прочитали и согласны с пользовательским соглашением http://www.mathnet.ru/rus/agreement

Параметры загрузки:

IP : 54.198 .55 .26

26 апреля 2023 г., $11: 28: 14$ 


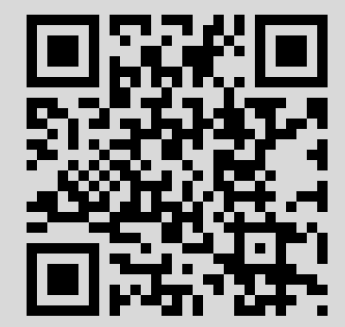




\section{О СПЕКТРАХ ЛИНЕЙНЫХ НЕПРЕРЫВНЫХ ОПЕРАТОРОВ НА СЕПАРАБЕЛЬНЫХ ПРОСТРАНСТВАХ ФРЕШЕ}

\section{C. А. Шкарин}

1. Формулировка основного результата. Напомним, что пространством Фреше (ПФ) [1] называется полное метризуемое локально выпуклое пространство. Все векторные пространства в этой работе предполагаются комплексными.

ТЕОРема 1. Пусть Т-линейный непрерывный оператор (далее ЛНО) на сепарабельном $\Pi \Phi X$. Тогда спектр [2] $\sigma(T)$ оператора $T$ имеет тип $G_{\delta \sigma}$ (определения дискриптивных типов множеств см. в [3]).

Теорема 1 доказывается в [4]. Мы даем ниже ее более короткое доказательство. Хорошо известны примеры [5], [6] ЛНО на сепарабельных ПФ, спектры которых некомпактны. Следующая теорема является основным результатом работы.

Tеорема 2. Пусть $A \subseteq \mathbb{C}$ - множсество типа $G_{\delta \sigma}$. Тогда существует бесконечномерное сепарабельное $\Pi \Phi X$ и ЛНО $т$ на $X$ такие, что $\sigma(T)=A$.

2. Доказательство теоремы 1. Пусть полунормы $p_{n}(n \in \mathbb{N})$ задают топлогию П $X$, $2 p_{n} \leqslant p_{n+1}$ для всех $n$ и $\left\{x_{n}: n \in \mathbb{N}\right\}-$ плотная в $X$ последовательность. Положим

$$
\begin{gathered}
A=\{z \in \mathbb{C}: \text { отображение }(T-z I): X \rightarrow(T-z I)(X) \text { является гомеоморфизмом }\} \\
B=\{z \in \mathbb{C}:(T-z I)(X) \text { не плотно в } X\} .
\end{gathered}
$$

Легко видеть, что

$$
\begin{gathered}
\sigma(T)=B \cup(\mathbb{C} \backslash A), \\
B=\bigcup_{m, n \in \mathbb{N}} \bigcap_{y \in X} B_{m, n, y}, \quad \text { где } B_{m, n, y}=\left\{z \in \mathbb{C}: p_{m}\left(T y-z y-x_{n}\right) \geqslant 1\right\} \\
A=\bigcap_{n \in \mathbb{N}} \bigcup_{m \in \mathbb{N}} \bigcap_{y \in X} A_{m, n, y}, \quad \text { где } A_{m, n, y}=\left\{z \in \mathbb{C}: p_{m}(T y-z y) \geqslant p_{n}(y)\right\} .
\end{gathered}
$$

Замкнутость множеств $B_{m, n, y}$ и $A_{m, n, y}$ вытекает из непрерьвности полунорм $p_{m}$. В силу $(2)$ и (3) множества $B$ и $A$ имеют тип $F_{\sigma}$ и $F_{\sigma \delta}$ соответственно. Согласно $(1)$ множество $\sigma(T)$ имеет тип $G_{\delta \sigma}$.

(C) С.А. ШКАРин

2001 
3. Доказательство теоремы 2. Пусть $M \subseteq \mathbb{C}$ - непустое множество типа $G_{\delta}$ и $\sup \{|z|$ : $z \in M\}<1$. Тогда $\mathbb{C} \backslash M=\bigcup_{n=0}^{\infty} A_{n}$, где множества $A_{n} \subseteq \mathbb{C}$ замкнуты, $A_{n} \subseteq A_{n+1}$ при всех $n$ и $\{z \in \mathbb{C}:|z| \geqslant 1\} \subseteq A_{0}$. Пусть $E_{M}$ - линейное пространство формальных выражений вида $\sum_{k=1}^{n} c_{k} \widehat{x}_{k}$, где $n \in \mathbb{N}, c_{k} \in \mathbb{C}$ и $x_{k} \in M$. Если $z \in \mathbb{C}$ и $f$-комплекснозначная функция, определенная в окрестности точки $z$, положим

$$
\operatorname{Lip}[f](z)=\lim _{\varepsilon \downarrow 0}(\inf \{c>0:|f(x)-f(y)|<c|x-y| \text { при }|x-z|<\varepsilon,|y-z|<\varepsilon\}) .
$$

Очевидно, что

$$
\begin{gathered}
\operatorname{Lip}[f+g](z) \leqslant \operatorname{Lip}[f](z)+\operatorname{Lip}[g](z), \quad \operatorname{Lip}[f g](z) \leqslant|f(z)| \operatorname{Lip}[g](z)+|g(z)| \operatorname{Lip}[f](z), \\
\operatorname{Lip}\left[\frac{f}{g}\right](z) \leqslant \frac{|f(z)| \operatorname{Lip}[g](z)+|g(z)| \operatorname{Lip}[f](z)}{|g(z)|^{2}} .
\end{gathered}
$$

Пусть $\mathscr{L}$ - пространство всех ограниченных липшицевых функций $\varphi: \mathbb{C} \rightarrow \mathbb{C}$ с нормой

$$
p(\varphi)=\max \left\{\sup _{z \in \mathbb{C}}|\varphi(z)|, \sup _{z \in \mathbb{C}} \operatorname{Lip}[\varphi](z)\right\} .
$$

В силу (5) $\varphi \psi \in \mathscr{L}$ для любых $\varphi, \psi \in \mathscr{L}$ и

$$
p(\varphi \psi) \leqslant 2 p(\varphi) p(\psi)
$$

Для $f: M \rightarrow \mathbb{C}$ и $\xi=\sum_{k=1}^{n} c_{k} \widehat{x}_{k} \in E_{M}$ положим

$$
\begin{gathered}
\langle\xi, f\rangle=\sum_{k=1}^{n} c_{k} f\left(x_{k}\right), \quad T_{f} \xi=\sum_{k=1}^{n} c_{k} f\left(x_{k}\right) \widehat{x}_{k}, \\
\|\xi\|_{f}=\sup \{|\langle\xi, f \varphi\rangle|: \varphi \in \mathscr{L}, p(\varphi) \leqslant 1\} .
\end{gathered}
$$

Ясно, что $\langle\cdot, f\rangle$ - линейный функционал, $T_{f}$ - линейный оператор и $\|\cdot\|_{f}$ - полунорма на $E_{M}$. Нетрудно проверить, что

$$
\left\langle T_{f} \xi, g\right\rangle=\langle\xi, f g\rangle \quad \text { и } \quad T_{f}\left(T_{g} \xi\right)=T_{f g} \xi .
$$

Лемма 1. Ecлu $a, f, g: M \rightarrow \mathbb{C}, b \in \mathscr{L}, \xi \in E_{M} u a(z) f(z)=b(z) g(z)$ npu всеx $z \in M$, mo $\left\|T_{a} \xi\right\|_{f} \leqslant 2 p(b)\|\xi\|_{g}$.

ДОКАЗАТЕЛЬСТво. В силу (6)-(8) имеем

$$
\begin{aligned}
\left\|T_{a} \xi\right\|_{f} & =\sup \left\{\left|\left\langle T_{a} \xi, f \varphi\right\rangle\right|: \varphi \in \mathscr{L}, p(\varphi) \leqslant 1\right\}=\sup \{|\langle\xi, g b \varphi\rangle|: \varphi \in \mathscr{L}, p(\varphi) \leqslant 1\} \\
& \leqslant \sup \{|\langle\xi, g \psi\rangle|: \psi \in \mathscr{L}, p(\psi) \leqslant 2 p(b)\} \leqslant 2 p(b)\|\xi\|_{g} .
\end{aligned}
$$

Пусть теперь $f_{n}(z)=\inf \left\{|z-x|: x \in A_{n}\right\}$. Очевидно, что $f_{n} \in \mathscr{L}$ и $p\left(f_{n}\right) \leqslant 1$. Наделим $E_{M}$ топологией $\tau$, задаваемой нормами

$$
\|\xi\|_{k, m}=\|\xi\|_{g_{k, m}}, \quad \text { где } g_{k, m}(z)=\left(f_{k}(z)\right)^{-m}, \quad k, m \in \mathbb{Z}_{+}=\{j \in \mathbb{Z}: j \geqslant 0\} .
$$

Лемма 2. Отображсение $x \mapsto \widehat{x}$ из $M$ в $\left(E_{M}, \tau\right)$ непрерывно.

ДоказАтельство. Пусть $x_{n} \in M(n \in \mathbb{N}), \lim _{n \rightarrow \infty} x_{n}=x \in M, k, m \in \mathbb{Z}_{+}, \varphi \in \mathscr{L}$, $p(\varphi) \leqslant 1$. Тогда $\left|x_{n}-x\right|<\varepsilon=f_{k}(x) / 2$ при достаточно больших $n$ (далее мы будем рассматривать только такие $n$ ). Если $y \in M,|y-x|<\varepsilon$, то $\varepsilon<f_{k}(y)<3 \varepsilon$ и согласно (5) $\operatorname{Lip}\left[\varphi g_{k, m}\right](y) \leqslant c=m 3^{m-1} \varepsilon^{-m-1}+\varepsilon^{-m}$. Тогда в силу $(7)\left|\left\langle\widehat{x}-\widehat{x}_{n}, \varphi g_{k, m}\right\rangle\right| \leqslant c\left|x_{n}-x\right|$. Значит, $\left\|\widehat{x}_{n}-\widehat{x}\right\|_{k, m} \leqslant c\left|x_{n}-x\right|$ и $\widehat{x}_{n}$ сходится к $\widehat{x}$ в $\left(E_{M}, \tau\right)$.

Лемма 3. Пусть $\xi \in E_{M}, n, k, m \in \mathbb{Z}_{+} u n \geqslant k$. Тогдa $\|\xi\|_{k, m} \leqslant(4 m+2)\|\xi\|_{n, m+1}$. 
ДоКАЗАТЕльство. Пусть $b: \mathbb{C} \rightarrow \mathbb{C}, b(z)=0$ при $f_{k}(z)=0$ и $b(z)=f_{n}^{m+1}(z) f_{k}^{-m}(z)$ при $f_{k}(z) \neq 0$. В силу (5) $b \in \mathscr{L}$ и $p(b) \leqslant 2 m+1$. Требуемое неравенство теперь следует из леммы $1 \mathrm{c}$ $a=1, f=g_{k, m}, g=g_{n, m+1}$.

Пусть $h: \mathbb{C} \rightarrow \mathbb{C}, h(z)=z$ при $|z|<1$ и $h(z)=z /|z|$ при $|z| \geqslant 1$. Очевидно, $h \in \mathscr{L}$ и $p(h)=1$.

Лемма 4. Пусть $\xi \in E_{M} u k, m \in \mathbb{Z}_{+}$. Тогдa $\left\|T_{h} \xi\right\|_{k, m} \leqslant 2\|\xi\|_{k, m}$.

ДоКАЗАТЕЛЬСтво. Достаточно применить лемму 1 с $b=a=h$ и $f=g=g_{k, m}$.

Лемма 5. Пусть $k, n, m \in \mathbb{Z}_{+}, z \in A_{k}, n \geqslant k$. Тогда оператор $\left(T_{h}-z I\right): E_{M} \rightarrow E_{M}$ биективен $и\left\|\left(T_{h}-z I\right)^{-1} \xi\right\|_{n, m} \leqslant 6\|\xi\|_{n, m+2}$.

ДоКАЗАТЕЛЬСТво. Пусть $a: M \rightarrow \mathbb{C}, a(x)=(x-z)^{-1}$. В силу (8)

$$
\left(T_{h}-z I\right) T_{a}=T_{a}\left(T_{h}-z I\right)=I
$$

Следовательно, оператор $T_{h}-z I$ обратим с обратным $T_{a}$. Пусть $b: \mathbb{C} \rightarrow \mathbb{C}, b(x)=f_{n}^{2}(x) /(x-z)$ при $x \neq z, b(z)=0$. В силу (5) $\operatorname{Lip}[b](x) \leqslant\left(2 f_{n}(x)|x-z|+f_{n}^{2}(x)\right)|x-z|^{-2}$ при $x \neq z$. Учитьвая, что $z \in A_{k} \subseteq A_{n}$, получаем неравенство $f_{n}(x) \leqslant|x-z|$. Значит, $\operatorname{Lip}[b](x) \leqslant 3$. Кроме того, $b(x) \leqslant f_{n}(x) \leqslant 1$. Следовательно, $b \in \mathscr{L}$ и $p(b) \leqslant 3$. Требуемое неравенство следует из леммы $1 \mathrm{c}$ $f=g_{n, m}, g=g_{n, m+2}$.

ЛЕмма 6. Для всякого непустого ограниченного множества $M \subseteq \mathbb{C}$ типа $G_{\delta}$ существуют сепарабельное ПФ $X$ и ЛНО $Т: X \rightarrow X$ такие, что $\sigma(T)=M$.

ДокаЗАТЕЛЬСтво. Можно считать, что $\sup \{|z|: z \in M\}<1$. Пусть $X$ - пополнение метризуемого локально выпуклого пространства $\left(E_{M}, \tau\right)$. По лемме $2 \Pi \Phi X$ сепарабельно как замыкание линейной оболочки сепарабельного множества $\{\widehat{x}: x \in M\}$. По лемме 4 оператор $T_{h}: E_{M} \rightarrow E_{M}$ непрерьвен и, следовательно, продолжается до ЛНО $T: X \rightarrow X$. Пусть $z \in \mathbb{C} \backslash M$, $k, m \in \mathbb{Z}_{+}$. Выберем $n \in \mathbb{Z}_{+}$такое, что $z \in A_{n}$. Биективность оператора $\left(T_{h}-z I\right): E_{M} \rightarrow E_{M}$ следует из того, что $h(x)-z \neq 0$ при всех $x \in M$. Пусть $\xi \in E_{M}$. Согласно лемме 3

$$
\left\|\left(T_{h}-z I\right)^{-1} \xi\right\|_{k, m} \leqslant(4 m+2)\left\|\left(T_{h}-z I\right)^{-1} \xi\right\|_{l, m+1},
$$

где $l=\max \{k, n\}$. Согласно лемме $5\left\|\left(T_{h}-z I\right)^{-1} \xi\right\|_{l, m+1} \leqslant 6\|\xi\|_{l, m+3}$. Из последних двух неравенств получаем $\left\|\left(T_{h}-z I\right)^{-1} \xi\right\|_{k, m} \leqslant 12(2 m+1)\|\xi\|_{l, m+3}$. Таким образом, оператор $\left(T_{h}-z I\right)^{-1}: E_{M} \rightarrow E_{M}$ непрерьвен и, значит, продолжается до ЛНО $S: X \rightarrow X$. Операторы $S(T-z I)$ и $(T-z I) S$ действуют тождественно на плотном множестве $E_{M}$. Значит, $S(T-z I)=$ $(T-z I) S=I$ и $z \notin \sigma(T)$. Таким образом, $\sigma(T) \subseteq M$. Если же $x \in M$, то в силу (7) $T \widehat{x}=T_{h} \widehat{x}=$ $h(x) \widehat{x}=x \widehat{x}$. Поэтому $x \in \sigma(T)$. Следовательно, $\sigma(T)=M$.

Перейдем к доказательству теоремы 2.

Случай 1. Множество $A=\varnothing$. Пространство $X=\left\{\varphi \in C^{\infty}[0,1]: f^{(j)}(0)=0\right.$ при всех $\left.j \geqslant 0\right\}$ с топологией равномерной сходимости всех производных является [7] бесконечномерным сепарабельным ПФ, причем оператор дифференцирования $T(f)=f^{\prime}$ непрерьвен и имеет пустой спектр.

Случай 2. Множество $A$ непусто и конечно. Надо взять прямую сумму оператора из случая 1 и оператора на конечномерном пространстве со спектром $A$.

Случай 3. Множество $A$ бесконечно. Представим $A$ в виде объединения последовательности $A_{n}$ ограниченных непустых множеств типа $G_{\delta}$. По лемме 6 для каждого $n \in \mathbb{N}$ найдется сепарабельное ПФ $X_{n}$ и ЛНО $T_{n}$ такие, что $\sigma\left(T_{n}\right)=A_{n}$. Пусть $X=\prod_{n=1}^{\infty} X_{n}, T: X \rightarrow X$, $(T x)_{n}=T_{n} x_{n}$. Тогда $X$ - бесконечномерное сепарабельное ПФ и оператор $T$ непрерьвен. Легко видеть, что $\sigma(T)=\bigcup_{n=1}^{\infty} A_{n}=A$. 
4. Нерешенные проблемы. Сушествует ряд нерешенных проблем, связанных с темой этой работы. Перечислим некоторые из них.

ПроБлема 1. Можно ли избавиться от требования сепарабельности в теореме 1 ?

ПроБЛЕма 2 (Е.А. Горин). Пусть $X$ - такое ПФ, что спектр любого ЛНО на $X$ ограничен. Верно ли, что пространство $X$ нормируемо?

ПроБлема 3 . Пусть $A \subseteq \mathbb{C}$ - множество типа $G_{\delta \sigma}$. Существует ли ядерное [7] ПФ $X$ и ЛНО $T$ на $X$ такие, что $\sigma(T)=A$ ?

Автор благодарен О.Г. Смолянову и А. И. Штерну за внимание к работе.

\section{СПИСОК ЦИТИРОВАННОЙ ЛИТЕРАТУРЫ}

1. Робертсон А., Робертсон В. Топологические векторные пространства. М.: Мир, 1967. 2. Данфорд Н., Шварц Т. Линейные операторы. Т. 1. М.: Мир, 1962. 3. Натансон И. П. Теория функций вещественной переменной. М.: Наука, 1974. 4. Slodowski S. // Proc. Roy. Soc. Edinb. Sect. 1981. V. 90. № 3-4. P. 257-261. 5. Lien P., Wong M. W. // Math. Japon. 1995. V. 41. №1. P. 169-173. 6. Wong M. W. // Math. Japon. 1993. V. 38. №4. P. 719-722. 7. Пич А. Ядерные локально выпуклые пространства. М.: Мир, 1969.

Московский государственный университет им. М.В. Ломоносова

Поступило

E-mail: stas@graal.math.msu.su

08.04.1999

Исправленный вариант

21.07.2000 